\title{
Odorant Binding Changes the Electrical Properties of Olfactory Re- ceptors at the Nanoscale
}

\author{
Anna Lagunas*, Christine Belloir, Loïc Briand, Pau Gorostiza, Josep Samitier* \\ Anna Lagunas - Biomedical Research Networking Center in Bioengineering, Biomaterials, and Nanomedicine \\ (CIBER-BBN), 28029 Madrid, Spain; Institute for Bioengineering of Catalonia (IBEC), Barcelona Institute of Science \\ and Technology, 08028 Barcelona, Spain. \\ Christine Belloir - Centre des Sciences du Goût et de l'Alimentation, AgroSup Dijon, CNRS, INRAE, Université \\ Bourgogne Franche-Comté, Dijon, France.
}

Loïc Briand - Centre des Sciences du Goût et de l'Alimentation, AgroSup Dijon, CNRS, INRAE, Université Bourgogne Franche-Comté, Dijon, France.

Pau Gorostiza - Institute for Bioengineering of Catalonia (IBEC), Barcelona Institute of Science and Technology, 08028 Barcelona, Spain; Biomedical Research Networking Center in Bioengineering, Biomaterials, and Nanomedicine (CIBER-BBN), 28029 Madrid, Spain; Catalan Institution for Research and Advanced Studies (ICREA), Barcelona o8o11, Spain.

Josep Samitier - Institute for Bioengineering of Catalonia (IBEC), Barcelona Institute of Science and Technology, o8028 Barcelona, Spain; Biomedical Research Networking Center in Bioengineering, Biomaterials, and Nanomedicine (CIBER-BBN), 28029 Madrid, Spain; Department of Electronics and Biomedical Engineering, University of Barcelona (UB), o8028 Barcelona, Spain.

Supporting Information Placeholder

\begin{abstract}
The transduction of odorant binding into cellular signaling by olfactory receptors (ORs) is not understood and knowing its mechanism would enable developing new pharmacology and biohybrid electronic detectors of volatile organic compounds bearing high sensitivity and selectivity. The electrical characterization of ORs in bulk experiments is subject to microscopic models and assumptions. We have directly determined the nanoscale electrical properties of ORs immobilized in a fixed orientation, and their change upon odorant binding, using electrochemical scanning tunneling microscopy (EC-STM) in near-physiological conditions. Recordings of current versus time, distance, and electrochemical potential allows determining the OR impedance parameters and their dependence with odorant binding. Our results allow validating OR structural-electrostatic models and their functional activation processes.
\end{abstract}

Olfactory receptors (ORs) comprise the largest multigene family in the vertebrates, with about 400 genes identified in humans.,2 They are expressed primarily by olfactory sensory neurons located in the olfactory epithelium in the nasal cavity and are responsible for odorant detection. Moreover, the expression of ORs in other tissues have been reported, where they exert distinct biological functions.3,4 ORs belong to the class A (rhodopsin-like) family of $G$ protein-coupled receptors (GPCRs). Around half of the GPCRs are ORs. GPCRs are the most abundant membrane proteins having widespread, significant roles in signal transduction in cells. Therefore, they are a major pharmacological target, with approximately the $40 \%$ of approved drugs on the market targeting GPCRs. 5,6

The mechanism of olfactory transduction in the main olfactory epithelium involves OR switching from a conformationally inactive state towards an active state upon ligand binding, which couples with the intracellular $G$ protein $G_{\text {olf }}$ and activates adenylyl cyclase type-III. This leads to an increase of the intraciliary level of cyclic adenosine monophosphate (cAMP) opening cyclic-nucleotide-gated (CNG) non-selective cation channels. The subsequent ionic flux causes membrane depolarization, the activation of $\mathrm{Na}^{+}$-channels and consequently, action potential firing. ${ }^{7}$ ORs show high selectivity and sensitivity towards odorant detection, ${ }^{8}$ a characteristic that prompted the development of biohybrid sensors using ORs for the detection of volatile organic compounds.9,10 This made the electrical characterization of ORs a subject of interest, which has been mainly addressed by electrochemical impedance spectroscopy (EIS) in bulk experiments. ${ }^{11,12}$ However, their microscopic interpretation relies on theoretical models of the protein electric properties and is subject to assumptions. These models are generally based on equivalent impedance networks trying to link the dipoles and charges of the backbone and side chains of the receptor in its native and activated state, with the experimental results obtained.13,14 Here, we have determined directly the nanoscale electrical properties of human OR hORıAı in the presence of its cognate ligand dihydrojasmone, using electrochemical scanning tunneling 
microscopy (EC-STM) under bipotentiostatic control. In particular, we have measured current-voltage $(I-V)$, current-time $(I-t)$ and current-distance $(I-z)$ characteristics of the receptor immobilized in a fixed orientation in a near-physiological environment. ORs behave as parallel resistor-capacitor (RC) circuits with $\mathrm{R}$ and $\mathrm{C}$ decreasing by $20 \%$ upon ligand binding. Our results will allow validating OR structural-electrostatic models and their functional activation including odorant binding at the extracellular side and guanosine triphosphate (GTP) binding and G protein release at the intracellular side.

$\mathrm{hOR}_{1} \mathrm{~A}_{1}$ was overexpressed in a stable tetracycline inducible HEK293S GnTI- cell line as previously described.15 The receptor was engineered by inserting the epitope tags rhoiD 4 at the C-terminal and FLAG at the N-terminal to allow its purification and detection. Circular dichroism analysis demonstrated that detergent-solubilized FLAG-rhoiD4-tagged hORıAıwas properly folded into $\alpha$-helical structure, as expected for the secondary structures of a GPCR. ${ }^{16}$ Receptor functionality was assessed by ligand binding using an intrinsic tryptophan fluorescence assay, which revealed the receptor bound its cognate odorant, dihydrojasmone, with an affinity in the micromolar range exhibiting a $K_{d}$ value of $1.4 \pm 0.5 \mu \mathrm{M}$ (Figure $1 \mathrm{~A}$ ).

A.

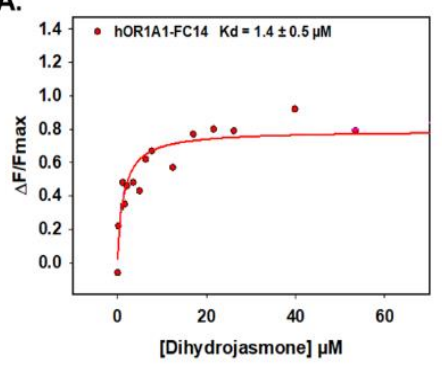

B.

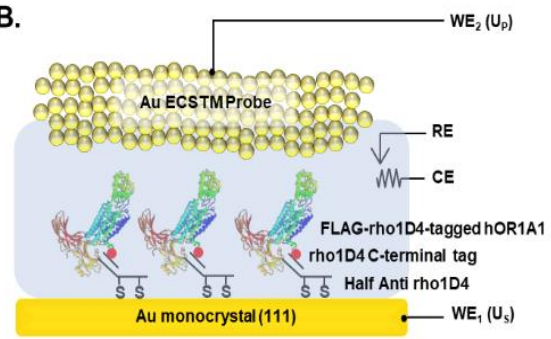

Figure 1. (A) Binding activity of purified hORıAı using intrinsic tryptophan fluorescence. Dose-response relationship of hORıAı fluorescence $(\lambda$ exc $=280 \mathrm{~nm}, \lambda$ exc $=340 \mathrm{~nm})$ following dihydrojasmone application. The data were fitted with sigmoid dose-response curves and $K_{d}$ value obtained (mean \pm s.d, $\mathrm{N}=3$ ). (B) Schematic representation of the experimental setup. Due to lack of any crystalline structure for hOR $1 A_{1}$, sequence homology with Phyrez has been used for the receptor representation. Abbreviations: WE, working electrode; RE, reference electrode; $\mathrm{CE}$, counter electrode.

We imaged by atomic force microscopy (AFM) and measured the single-protein current-bias voltage $(I-V)$ characteristics of $\mathrm{hOR}_{1} \mathrm{~A}_{1}$ in a physiological environment $(50 \mathrm{mM}$ phosphate buffer $\mathrm{pH}$ 7.4) by EC-STM. The experiments were conducted under bipotentiostatic control of the probe and sample electrodes versus an $\mathrm{Ag} / \mathrm{AgCl}$ (SSC) reference electrode, with and without the presence of dihydrojasmone. $I-V$ measurements were performed at a fixed separation between the probe and sample (given by a setpoint current of $0.4 \mathrm{nA}$ ) to avoid physical contact between the STM probe and the protein. ${ }^{17}$ The

STM feedback loop allowed fixing the setpoint current and it was turned off during $0.86 \mathrm{~s}$ to perform each $I-V$ recording at a rate of $581 \mathrm{mV} / \mathrm{s}$. This avoids the biasing that might be introduced by the contact geometry in contact mode, ${ }^{18}$ and provides a more flexible configuration.

A.

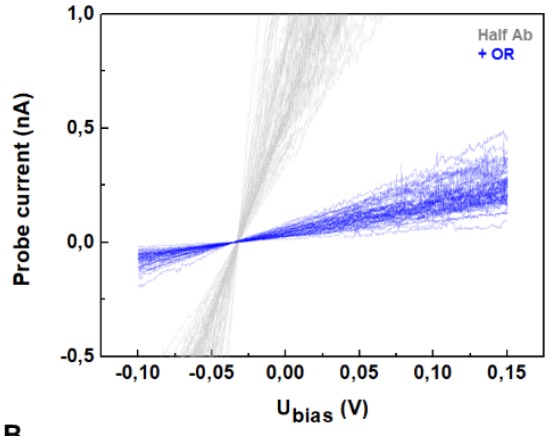

B.

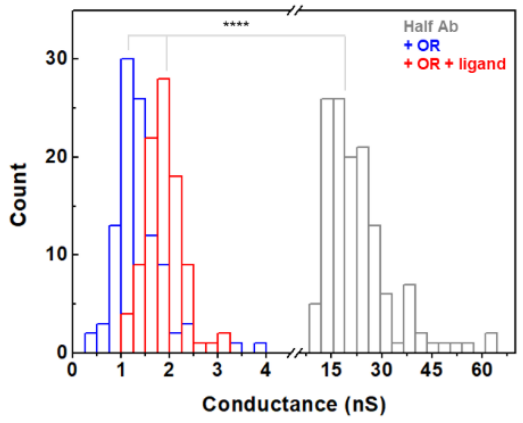

C.

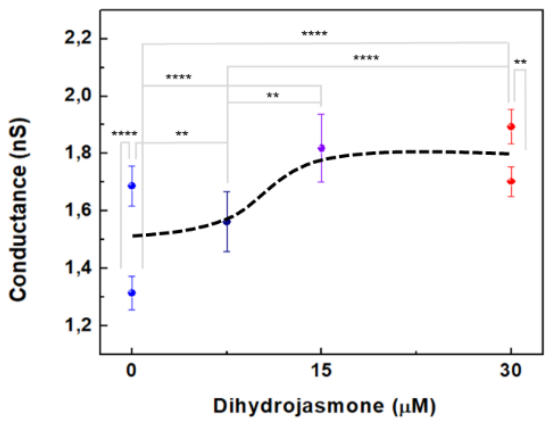

Figure 2. (A) Ensemble of EC-STM current-bias voltage ( $I-V)$ curves obtained for half anti-Rhodopsin (grey) and hORıAl (blue), showing the decrease in conductance upon receptor immobilization. (B) Conductance histograms obtained from the linear fitting of individual $I$ - $V$ curves for half anti-Rhodopsin (grey), hORıAı before (blue) and after incubation with 30 $\mu \mathrm{M}$ dihydrojasmone (red). (C) Plot of the conductance variation in hORıAıwith the increasing dihydrojasmone concentration. Dashed black lines are an eye-guide. Values are the mean \pm SE. $\mathrm{n}=150 .{ }^{*} \mathrm{P}<0.03,{ }^{* *} \mathrm{P}<0.01,{ }^{* * * *} \mathrm{P}<0.0001$. Experiments were conducted at a constant sample potential $\left(\mathrm{U}_{\mathrm{S}}\right)$ of $250 \mathrm{mV}$, current set point $=0.4 \mathrm{nA}, 50 \mathrm{mM}$ sodium phosphate buffer, $\mathrm{pH} 7.4$.

A uniform orientation of the receptor is essential in single molecule experiments, ${ }^{19,20}$ and to achieve it hORıA1 was immobilized on the Au(111) electrode of the EC-STM by half antiRhodopsin antibody, against the C-terminal tag Rho1D4, ${ }^{21}$ (Figure $1 \mathrm{~B}$ ). Coverage of the substrate was monitored by atomic force microscopy imaging (Figure $\mathrm{S}_{1}$ ). Height 
histograms show a peak centered at $4.8 \pm 0.9 \mathrm{~nm}$ after the incubation with the hORıAl, which was attributed to the receptor, in agreement with the sequence homology data obtained for hORıAl. ${ }^{22}$

Sample potential $\left(\mathrm{U}_{\mathrm{S}}\right)$ and initial probe potential (initial $\mathrm{U}_{\mathrm{P}}$ ) were set at $0.25 \mathrm{~V}$ and $0.45 \mathrm{~V}$, respectively. Faradaic leakage current was maintained below a few pA through probe insulation. ${ }^{23}$ To obtain each $I-V$ plot, the probe was positioned over the sample at a current set point of $0.4 \mathrm{nA}$, the feedback loop disconnected and the probe current recorded while a voltage ramp is applied to the EC-STM probe. Up to $150 I$ - $V$ curves were recorded at different sample positions by sweeping the probe potential back and forth from 0.15 to $0.40 \mathrm{~V}$ (bias $\left(\mathrm{U}_{\text {bias }}\right.$ $\left.=\mathrm{U}_{\mathrm{P}}-\mathrm{U}_{\mathrm{S}}\right)=-0.10$ to $\left.0.15 \mathrm{~V}\right)$, covering a physiological range of cell membrane potential. ${ }^{24}$

$I-V$ measurements of half anti-Rhodopsin antibody alone, and hORıAl immobilized through half anti-Rhodopsin antibody are shown in Figure 2A. The presence of hORıAl caused a pronounced reduction of conductance. We observed a linear $I-V$ response in all cases; thus, a single conductance value (fitted $I$ - $V$ slope) can be assigned to each $I-V$ trace. Figure $2 \mathrm{~B}$ shows measured distributions of the conductance values obtained for anti-Rhodopsin antibody, and hORıAı immobilized through half anti-Rhodopsin antibody with and without the presence of the OR cognate ligand dihydrojasmone at $30 \mu \mathrm{M}$ concentration. The presence of the ligand caused a significant increase of conductance in hORıAl.

To exclude contributions from the media in the gap between the probe and the sample, we conducted static break junction experiments. ${ }^{23,25}$ With the feedback loop transiently disconnected, we recorded current vs. time $(I-t)$ at a constant bias of $0.2 \mathrm{~V}\left(\mathrm{U}_{\mathrm{S}}=0.25 \mathrm{~V}\right.$ and $\left.\mathrm{U}_{\mathrm{P}}=0.45 \mathrm{~V}\right)$ and at a current set point of $0.3 \mathrm{nA}$. Spontaneous contact between the probe and the sample results in jumps (blinks) of the current that last as much as the contact does (Figure $\mathrm{S}_{2} \mathrm{~A}$ ). They represent solely the net conductance flowing through the receptor, thus without the contribution of the media. By collecting the blinks with subtracted current baseline and setting them to a common time origin, 2D-blinking maps were built. These maps show the variability in conductance that is introduced by the geometry of the contact (Figure $\mathrm{S}_{2} \mathrm{~B}$ ). Taking the most probable conductance peak, we observed that binding to dihydrojasmone caused a conductance increase (Figure $\mathrm{S}_{2} \mathrm{C}$ ) as in $\mathrm{I}-\mathrm{V}$ non-contact measurements. In general, conductance values obtained in $I-t$ measurements are lower, as expected from the more resistive environment of the protein.

Treatment with increasing concentrations of dihydrojasmone (o-3o $\mu \mathrm{M}$ ) lead to a growth of conductance (Figure $2 \mathrm{C}$ ). Fitting the data using Hill sigmoidal equation with variable slope (i.e., 4 parameters logistical) (Figure $S_{3}$ ) yielded an $\mathrm{EC}_{50}$ of $11.2 \mu \mathrm{M}$, in agreement with previous results, ${ }^{15}$ and thereby validating our EC-STM set-up for studying hORıA1-dihydrojasmone binding. A Hill coefficient of 9.9 was obtained from the fit indicating a non-linear relation between de EC-STM response and odorant concentration. Supralinear relation (i.e., Hill coefficient $>1$ ) has been previously reported for ORs and attributed to repeated binding. ${ }^{26,27}$

We also conducted current-distance $(\mathrm{I}-\mathrm{z})$ measurements on $\mathrm{hOR}_{1} \mathrm{~A}_{1}$ in the absence/presence of dihydrojasmone at the same concentrations used in $I-V$ experiments (Figure $\mathrm{S}_{4}$ ). $I-z$ measurements were conducted as previously described, 19,28 at a current set point of $0.4 \mathrm{nA}$, and at a constant bias of $0.2 \mathrm{~V}$ $\left(\mathrm{U}_{\mathrm{S}}=0.25 \mathrm{~V}\right.$ and $\left.\mathrm{U}_{\mathrm{P}}=0.45 \mathrm{~V}\right)$. Up to $100 \mathrm{I}$ - $\mathrm{Z}$ curves were recorded per sample and distance decay factors $(\beta)$ were quantified from individual semi-logarithmic $I$-z plots. We observed that $\beta$ decreases with the increasing dihydrojasmone concentration, suggesting that dihydrojasmone increases the spatial span of the hOR1A1 currents.

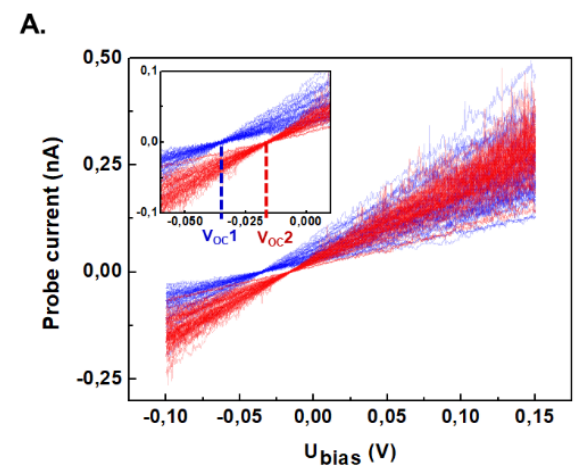

B.

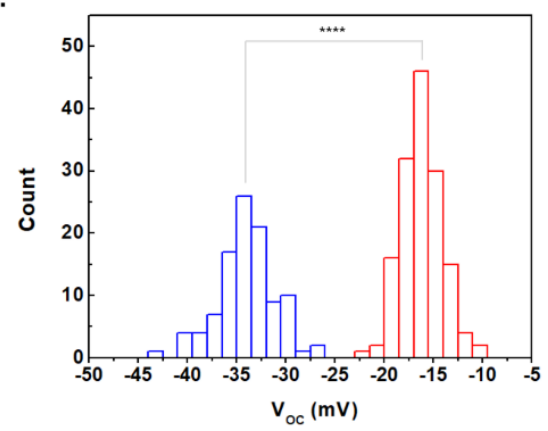

C.

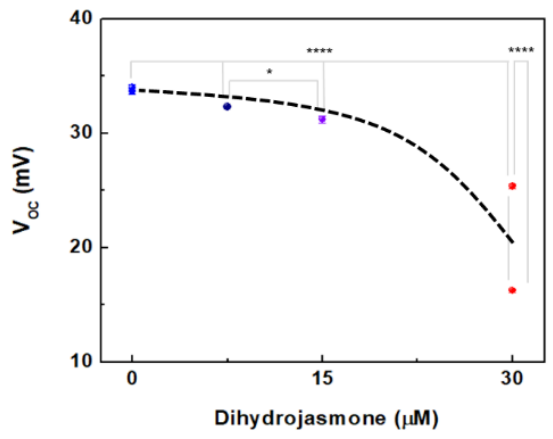

Figure 3. (A) Superimposition of $I-V$ curves from hORıA1 before (blue) and after (red) incubation with $30 \mu \mathrm{M}$ dihydrojasmone ligand. Dihydrojasmone causes a shift towards a lower absolute value of the open-circuit voltage $\left(\mathrm{V}_{\mathrm{OC}}\right)$, inset. (B) $V_{O C}$ histograms obtained from the linear fitting of individual $I$ - $V$ curves in A. (C) Plot of the $V_{O C}$ variation (absolute values) in hORıAlwith the increasing dihydrojasmone concentration. Dashed black lines are an eye-guide. Values are the mean \pm SE. $\mathrm{n}=150 .{ }^{*} \mathrm{P}<0.03,{ }^{* *} \mathrm{P}<0.01,{ }^{* * * *} \mathrm{P}<0.0001$. Experiments were conducted at a constant sample potential $\left(U_{S}\right)$ of $250 \mathrm{mV}$, current set point $=0.4 \mathrm{nA}, 50 \mathrm{mM}$ sodium phosphate buffer, $\mathrm{pH} 7 \cdot 4$.

Besides the conductance increase, $I-V$ curves showed a shift of around $20 \mathrm{mV}$ in the open-circuit voltage $\left(\mathrm{V}_{\mathrm{OC}}\right)$ towards lower 
potentials (absolute values) in the presence of dihydrojasmone (Figure $3 \mathrm{~A}$ ), which is not observed in the absence of the ligand (Figure $\mathrm{S}_{5} \mathrm{~A}$ ). The $\mathrm{V}_{\mathrm{OC}}$ value obtained from the $I-V$ curves for the hOR1A1 was $33.8 \pm 0.2 \mathrm{mV}$, which was similar to that obtained for the half anti-Rhodopsin antibody alone ( $\mathrm{V}_{\mathrm{OC}}$ $=31.5 \pm 0.2 \mathrm{mV})$ and significantly higher $(\mathrm{P}<0.0001)$ than that obtained for hOR1A1 with dihydrojasmone at $30 \mu \mathrm{M}$ concentration $\left(\mathrm{V}_{\mathrm{OC}}=21.8 \pm 0.3 \mathrm{mV}\right.$; Figure $\left.3 \mathrm{~B}\right)$. This indicates that ligand binding produces a decrease in the receptor potential. Treatment with increasing concentrations of dihydrojasmone (o-3o $\mu \mathrm{M})$ caused $\mathrm{V}_{\mathrm{OC}}$ to decrease exponentially with a 1/e of $12.8 \mu \mathrm{M}$ (Figure ${ }_{3} \mathrm{C}$ and $\mathrm{S}_{5} \mathrm{D}$ ).

The existence of a Voc different of zero in the experimental results suggests that $\mathrm{hOR}_{1} \mathrm{Al}_{1}$ acts as an electrical first order system to a voltage ramp. Reported EIS measurements in bulk showed that ligand binding in ORs can be monitored following the variation of the impedance spectra. Nyquist plots were fitted using a modified Randles equivalent circuit, in which the response of the receptor was essentially described by the impedance of the Rp-CPE parallel circuit. ${ }^{29}$ In agreement with that, we modeled the electrical behavior of hOR1A1 as an RC parallel circuit (Figure $4 \mathrm{~A}$ ), and empiric impedance values were directly determined, resulting in $R_{1}=583 \mathrm{M} \Omega, C_{1}=0.10$ $\mathrm{nF}(\mathrm{SI})$. The interaction of OR with dihydrojasmone produces a decrease of around an $11 \%$ of the resistive part, and around a $30 \%$ of the capacitive part of the electrical equivalent circuit (from $683 \mathrm{M} \Omega$ to $517 \mathrm{M} \Omega$, and from $0.10 \mathrm{nF}$ to $0.07 \mathrm{nF}$, respectively. See SI).

In conclusion, we have measured the nanoscale conductance of hOR $\mathrm{A}_{1}$ in nearly physiological conditions and we have found that dihydrojasmone binding to the receptor causes an increase in conductance that can be measured from both $I-V$ and $I-t$ recordings, and which is dose dependent. The $\mathrm{EC}_{50}$ of 11.2 $\mu \mathrm{M}$ determined is within the range of $\mathrm{EC}_{50}$ values previously reported for $\mathrm{hOR}_{1} \mathrm{Al}_{1},{ }^{15}$ thus indicating that changes in conductance are correlated with ligand binding. Strikingly, dihydrojasmone binding produces a shift in $\mathrm{V}_{\mathrm{OC}}$ towards lower potentials. This indicates that ligand binding induces a depolarization of the receptor that causes a decrease in the impedance, in good agreement with the microscopic model proposed by Alfinito et al. for ORs, which is based on the outcome of bulk experiments. ${ }^{11,30}$ Dihydrojasmone binding causes a conformational change in the receptor that may alter charge distribution facilitating charge transfer, as demonstrated by $I$ $z$ measurements, and thereby increasing conductance (Figure $\left.{ }_{4} \mathrm{~B}\right)$ and spatially extending (reducing) the $\beta$ decay rate. The dependence of both conductance and $\mathrm{V}_{\mathrm{OC}}$ with ligand concentration agrees with the proposed mechanism of activation of GPCRs, for which a complex series of conformationally stable intermediates have been described to evolve towards a more active state with the increasing ligand concentration. ${ }^{31}$ Finally, the simultaneous measurement of RC equivalent by means of the $\mathrm{V}_{\mathrm{OC}}$ potential allows increasing the electrical sensitivity at single receptor level for biosensing applications. The electric model and fit predicts that faster $I-V$ ramps would provide even larger $\mathrm{V}_{\mathrm{OC}}$ shifts and thus higher ligand sensitivity both for fundamental and sensing applications.

Overall, EC-STM proved as a reliable technique to study the nanoscopic electrical properties of $\mathrm{hOR}_{1} \mathrm{~A}_{1}$, which were measured with unprecedented control over the receptor orientation and in nearly physiological conditions. The changes induced by ligand binding in conductance and specially in $\mathrm{V}_{\mathrm{OC}}$ values, pave the way towards the development of better biohybrid odorant sensors.

A.
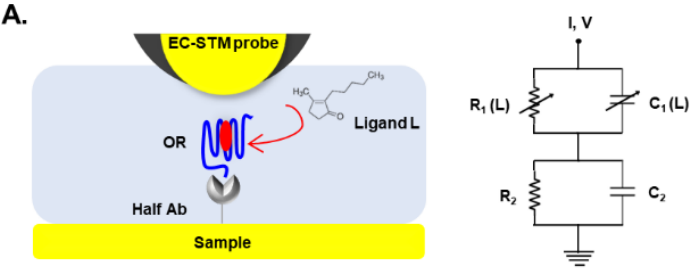

B.

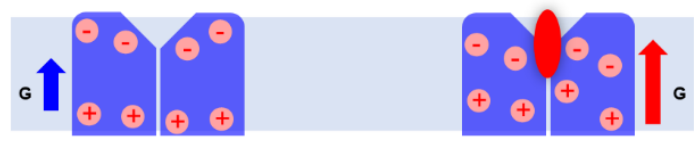

Figure 4. (A) Electrical equivalent model proposed: a combination of RC parallel circuits for the OR $\left(R_{1} C_{1}\right)$ and the half antibody $\left(R_{2} C_{2}\right)$. The values of $R_{1}$ and $C_{1}$ are regulated by the ligand concentration. (B) Dihydrojasmone binding to hORıAl may cause charge reorganization facilitating charge transfer and increasing conductance.

\section{ASSOCIATED CONTENT}

\section{Supporting Information}

The Supporting Information is available free of charge on the ACS Publications website.

Materials, sample preparation, details on EC-STM measurements, statistics, atomic force microscopy measurements, conductance fitting, $I-z$ and $I-t$ measurements, and $\mathrm{V}_{\mathrm{OC}}$ analysis (PDF)

\section{AUTHOR INFORMATION}

Corresponding Authors

Anna Lagunas - Biomedical Research Networking Center in Bioengineering, Biomaterials, and Nanomedicine (CIBER-BBN), 28029 Madrid, Spain; Institute for Bioengineering of Catalonia (IBEC), Barcelona Institute of Science and Technology (BIST), o8028 Barcelona, Spain.; E-mail: alagunas@ibecbarcelona.eu Josep Samitier - Institute for Bioengineering of Catalonia (IBEC), Barcelona Institute of Science and Technology (BIST), o8028 Barcelona, Spain; Biomedical Research Networking Center in Bioengineering, Biomaterials, and Nanomedicine (CIBER$B B N), 28029$ Madrid, Spain; Department of Electronics and Biomedical Engineering, University of Barcelona (UB), o8028 Barcelona, Spain; E-mail: jsamitier@ibecbarcelona.eu

\section{Notes}

The authors declare no competing financial interests.

\section{ACKNOWLEDGMENT}

This work was supported by the Biomedical Research Networking Center (CIBER), Spain. CIBER is an initiative funded by the VI National R\&D\&i Plan 2008-2011, Iniciativa Ingenio 2010, Consolider Program, CIBER Actions, and the Instituto de 
Salud Carlos III (RD16/ooo6/oo12; RD16/oo11/oo22), with the support of the European Regional Development Fund (ERDF).

This work was funded by the CERCA Program and by the Commission for Universities and Research of the Department of Innovation, Universities, and Enterprise of the Generalitat de Catalunya (2017-SGR-1079, 2017-SGR-1442 and 2017-SGR00465). This work was supported by grants from the Conseil Régional Bourgogne Franche-Comté (PARI grant) and the FEDER (European Funding for Regional Economical Development). This research also received funding from the European Union Research and Innovation Programme Horizon 2020
(Human Brain Project SGA3 No. 945539), DEEPER (ICT-362020-101016787), Agency for Management of University and Research Grants of the Government of Catalonia (CERCA Programme; 2017-SGR-1442 project; AGAUR, Clúster Emergent del Cervell Humà), and Ministry of Economy and Competitiveness (Grant PID2019-111493RB-Ioo). The authors also acknowledge Dr. Albert Cortijos Aragonés for help with $I-t$ measurements and data analysis. 


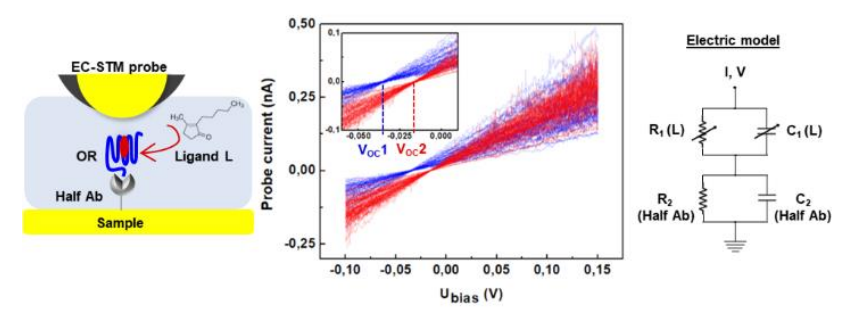

\section{REFERENCES}

${ }^{1}$ Buck, L.; Axel, R. A Novel Multigene Family May Encode Odorant Receptors: A Molecular Basis for Odor Recognition. Cell 1991, 65, 175-187.

2 Malnic, B.; Godfrey, P.A.; Buck, L.B. The Human Olfactory Receptor Gene Family. Proc. Natl. Acad. Sci. USA 2004, 101, 25842589.

${ }^{3}$ Feldmesser, E.; Olender, T.; Khen, M.; Yanai, I.; Ophir, R.; Lancet, D. Widespread Ectopic Expression of Olfactory Receptor Genes. BMC Genomics 2006, 7, 121.

4 Lee, S.-J.; Depoortere, I.; Hatt, H. Therapeutic Potential of Ectopic Olfactory and Taste Receptors. Nat. Rev. Drug Discov. 2019, 18, 116-138.

5 Hutchings, C. J.; Koglin, M.; Olson, W.C.; Marshall, F.H. Opportunities for therapeutic antibodies directed at G- protein-coupled receptors. Nat. Rev. Drug Discov. 2017, 16, 787-810.

${ }^{6}$ Hauser, A.S.; Attwood, M.M.; Rask-Andersen, M.; Schiöth, H.B.; Gloriam, D.E. Trends in GPCR Drug Discovery: New Agents, Targets and Indications Nat. Rev. Drug Discov. 2017, 16, 829-842.

7 Boccaccio, A. Patch-Clamp Recordings from Mouse Olfactory Sensory Neurons. In Olfactory Receptors. Methods and Protocols (Methods in Molecular Biology, 1820). Simoes de Souza, F. M., Antunes, G., Eds.; Humana Press, 2018. DOI: 10.1007/978-14939-8609-5.

${ }^{8}$ Bhandawat, V.; Reisert, J.; Yau, K.-W. Signaling by Olfactory Receptor Neurons Near Threshold. Proc. Natl. Acad. Sci. U.S.A. 2010, 107, 18682-18687.

9 Yang, H.; Kim, D.; Kim, J.; Moon, D.; Song, H.S.; Lee, M.; Hong, S.; Park, T.H. Nanodisc-Based Bioelectronic Nose Using Olfactory Receptor Produced in Escherichia coli for the Assessment of the Death-Associated Odor Cadaverine. ACS Nano 2017, 11, $11847-11855$.

${ }^{10}$ Yamada, T.; Sugiura, H.; Mimura, H.; Kamiya, K.; Osaki, T.; Takeuchi, S. Highly Sensitive VOC Detectors Using Insect Olfactory Receptors Reconstituted into Lipid Bilayers. Sci. Adv. 2021, 7, eabd2o13.

${ }^{11}$ Hou, X.; Jaffrezic-Renault, N.; Martelet, C.; Zhang, A.; Minic-Vidic, J.; Gorojankina, T.; Persuy, M.-A.; Pajot-Augy, E.; Salesse, R.; Akimov, V.; Reggiani, L.; Pennetta, C.; Alfinito, E.; Ruiz, O.; Gomila, G.; Samitier, J.; Errachid, A. A novel detection strategy for odorant molecules based on controlled bioengineering of rat olfactory receptor I 7 , Biosens. Bioelectron. 2007, 22, 1550-1555. ${ }^{12}$ Khadka, R.; Aydemir, N.; Carraher, C.; Hamiaux, C.; Colbert, D.; Cheema, J.; Malmström, J.; Kralicek, A.; Travas-Sejdic, J. An UltraSensitive Electrochemical Impedance-Based Biosensor Using Insect Odorant Receptors to Detect Odorants. Biosens. Bioelectron. 2019, 126, 207-2013.

${ }^{13}$ Alfinito, E.; Pousset, J.; Reggiani, L. PROTEOTRONICS: The Emerging Science of Protein-based Electronic Devices. J. Phys.: Conf. Ser. 2015, 647, 012002

${ }^{14}$ Alfinito, E.; Reggiani, L. Current-Voltage Characteristics of Seven-Helix Proteins from a Cubic Array of Amino Acids. Phys. Rev. E 2016, 93, 062401.

15 Belloir, C.; Miller-Leseigneur, M.-L.; Neiers, F.; Briand, L.; Le Bon, A.-M. Biophysical and Functional Characterization of the Human Olfactory Receptor ORıAı Expressed in a Mammalian Inducible Cell Line. Protein Expr. Purif. 2017, 129, 31-43

${ }^{16}$ Erdogmus, S.; Storch, U.; Danner, L.; Becker, J.; Winter, M.; Ziegler, N.; Wirth, A.; Offermanns, S.; Hoffmann, C.; Gudermann, T.; Mederos y Schnitzler, M. Helix 8 is the Essential Structural Motif of Mechanosensitive GPCRs. Nat. Commun. 2o19, 10, 5784 .

17 Artés, J.M.; López-Martínez, M.; Giraudet, A.; Díez-Pérez, I.; Sanz, F.; Gorostiza, P. Current-Voltage Characteristics and Transition Voltage Spectroscopy of Individual Redox Proteins. J. Am. Chem. Soc. 2012, 134, 20218-20221.

${ }^{18}$ Zhang, B.; Song, W.; Pang, P.; Lai, H.; Chen, Q.; Zhang, P.; Lindsay, S. Role of Contacts in Long-Range Protein Conductance. Proc. Natl. Acad. Sci. USA 2019, 26, 5886-5891.

${ }^{19}$ Lagunas, A.; Guerra-Castellano, A.; Nin-Hill, A.; Díaz-Moreno, I.; De la Rosa, M.A.; Samitier, J.; Rovira, C.; Gorostiza, P. Long Distance Electron Transfer through the Aqueous Solution between Redox Partner Proteins. Nat Commun. 2o18, 9, 5157. 
${ }^{20}$ Ortiz, M. L.; Zamora, R. A.; Giannotti, M. I.; Hu, C.; Croce, R.; Gorostiza, P. Distance and Potential Dependence of Charge Transport Through the P7oo Reaction Center of Photosystem I. ChemRxiv. Cambridge: Cambridge Open Engage 2o21; This content is a preprint and has not been peer-reviewed.

${ }^{21}$ Sharma, H.; Mutharasan, R. Half Antibody Fragments Improve Biosensor Sensitivity without Loss of Selectivity. Anal. Chem. 2013, 85, 2472-2477.

${ }^{22}$ Kelley, L.; Mezulis, S.; Yates, C.; Wass, M. N.; Sternberg, M. J. E. The Phyrez Web Portal for Protein Modeling, Prediction and Analysis. Nat. Protoc. 2015, 10, 845-858.

${ }^{23}$ Artés, J. M.; Díez-Pérez, I.; Gorostiza, P. Transistor-like Behavior of Single Metalloprotein Junctions. Nano Lett. 2012, 12 , 2679-2684.

${ }^{24}$ Kandel, E.R.; Schwartz, J.H.; Jessell, T.M. In Principles of Neural Science; McGraw-Hill 20oo; pp 1227-1246.

${ }_{25}$ Ruiz, M.P.; Aragonès, A.C.; Camarero, N.; Vilhena, J.G.; Ortega, M.; Zotti, L.A.; Pérez, R.; Cuevas, J.C.; Gorostiza, P.; DíezPérez, I. Bioengineering a Single-Protein Junction. J. Am. Chem. Soc. 2017, 139, 15337.

${ }^{26}$ Bhandawat,V.; Reisert, J.; Yau, K-W. Elementary Response of Olfactory Receptor Neurons to Odorants. Science 2005, 308, 1931-1934.

${ }_{27}$ Sanmartí-Espinal, M.; Iavicoli, P.; Calò, A.; Taulés, M.; Galve, R.; Marco, M.P.; Samitier, J. Quantification of Interacting Cognate Odorants with Olfactory Receptors in Nanovesicles. Sci. Rep. 2017, 7, 17483.

${ }^{28}$ Artés, J.M.; Díez-Pérez, I.; Sanz, F.; Gorostiza, P. Direct Measurement of Electron Transfer Distance Decay Constants of Single Redox Proteins by Electrochemical Tunneling Spectroscopy. ACS Nano 2011, 5, 2060.

29 Alfinito, E.; Pennetta, C.; Reggiani, L. Olfactory Receptor-Based Smell Nanobiosensors: An Overview of Theoretical and Experimental Results. SENSOR. ACTUAT. B-CHEM. 2010, 146, 554-558.

3o Alfinito, E.; Millithaler, J.F.; Reggiani, L.; Zine, N.; Jaffrezic-Renault, N. Human Olfactory Receptor 17-40 as an Active part of a Nanobiosensor: a Microscopic Investigation of its Electrical Properties. RSC Advances 2011, 1, 123-127.

${ }^{31}$ Kobilka, B. K.; Deupi, X. Conformational Complexity of G-Protein-coupled Receptors. Trends Pharmacol. Sci. 2oo7, 28, 397406. 